\title{
The Effect of Outdoor Weathering on Color Stability of Silicone and Acrylic Resin, Pigments-A Comparative Evaluation: An in vitro Study
}

\author{
${ }^{1}$ Lekha K, ${ }^{2} \mathrm{H}$ Dharanendra Kumar, ${ }^{3}$ Roseline Meshramkar, ${ }^{4}$ Ramesh K Nadiger
}

\begin{abstract}
Maxillofacial prosthetic materials either resin or elastomer used for the fabrication of facial prostheses have their own physical and mechanical properties. Maxillofacial prosthesis frequent replacement because the elastomer or acrylic resin and its pigments undergo color changes.

Purpose: Evaluate the effect of outdoor weathering on color stability of silicone and heat-cure acrylic with two different pigments.
\end{abstract}

Materials and methods: Total of 80 circular disk specimens, 40 silicone elastomer and 40 heat-cure clear acrylic resin were fabricated in the prepared mold and two dry earth pigments burnt sienna and cobalt blue was added to the specimens and it was subjected to simulated outdoor weathering. Before and after weathering, $\mathrm{CIE}$ values of both control and weathering groups were recorded by using spectrophotometer and tabulated. The obtained data were subjected to statistical analysis.

Results: Silicone elastomer with burnt sienna showed more color change when exposed to weathering followed by silicone with cobalt blue. Heat-cure with cobalt blue had shown some color change, heat-cure and burnt sienna were the most color stable.

Conclusion: From the study, it can be concluded that heat-cure acrylic resin with burnt sienna most color stable than silicone elastomer.

Keywords: Color stability, Dry earth pigments, Heat-cure acrylic, Maxillofacial prosthesis, Outdoor weathering, Silicone elastomer.

How to cite this article: Lekha K, Kumar HD, Meshramkar R, Nadiger RK. The Effect of Outdoor Weathering on Color Stability of Silicone and Acrylic Resin, Pigments-A Comparative Evaluation: An in vitro Study. Int J Prosthodont Restor Dent 2015;5(3):81-85.

\footnotetext{
${ }^{1}$ Professor and Head, ${ }^{2}$ Private Practitioner, ${ }^{3}$ Professor

${ }^{4}$ Professor and Dean (Support Services)

1,3,4 Department of Prosthodontics, SDM College of Dental Sciences and Hospital, Dharwad, Karnataka, India

${ }^{2}$ Private Practice, Muharraq Town, Al Muharraq, Bahrain

Corresponding Author: Lekha K, Professor and Head Department of Prosthodontics, SDM College of Dental Sciences and Hospital, Dharwad, Karnataka, India, Phone: 08362468142 e-mail: lekhakp@yahoo.com
}

\section{Source of support: Nil}

Conflict of interest: None

\section{INTRODUCTION}

The maxillofacial prosthetics currently finds itself experiencing more transformation than at any other time over the past 50 years of its recognized existence. Despite advances in plastic surgery, there will always be a need for maxillofacial prostheses for cancer and trauma patients.

The goal in maxillofacial prosthodontics is to restore the patient's appearance and protect the recession site allowing improvement in self esteem to help the patient to lead as normal life as possible. ${ }^{1}$

Maxillofacial prosthetic materials either resin or elastomer used for the fabrication of facial prostheses have their own physical and mechanical properties. Unfortunately, these materials demonstrate poor longterm durability, and a prosthesis may become torn or lose its color within a short period of time. This overall deterioration has been attributed to certain environmental factors, such as ${ }^{1}$ exposure to the ultraviolet component of natural sunlight, ${ }^{2}$ wetting and drying of the elastomer, ${ }^{3}$ and surface abrasion resulting from the application and removal of cosmetics. ${ }^{2,4-7}$ Unfortunately, little scientific attention has been accorded relative to constructive pigmentation to desire lifelike cosmetic realism. This involves exacting replication of subdermal, commonly referred to as intrinsic coloration and external or extrinsic coloration.

The discoloration of a facial prosthesis may be caused by color change of intrinsic or extrinsic coloration as a result of external environmental factors and is related to the intrinsic color stability of elastomer and colorants (pigments, flocking), or loss of external coloration on the prosthesis. Regardless of the type of elastomer used in the fabrication of a facial prosthesis, color degradation often is the cause of concern for patient as well as clinician. Different colorants and elastomer combinations are used to improve the color stability. Addition of colorants can have a stabilizing effect on color of elastomer and acrylic polymer when exposed to outdoor weathering. 
Its service life is usually 6 months to 2 years, with an average time of 10 to 12 months. ${ }^{8}$ This in vitro study was conducted to evaluate the effect of outdoor weathering on color stability of silicon and heat-cure acrylic resin with dry earth pigment, burnt sienna and cobalt blue and to compare the color stability of silicone and heatcure acrylic resin when exposed to outdoor weathering for 30 days. Before weathering CIE Lab values of both control and study groups were recorded using spectrophotometer and tabulated.

\section{MATERIALS AND METHODS}

The material tested was platinum silicone elastomer room temperature vulcanization (RTV) (Factor II, Inc), heatcure clear acrylic resin Dental Product of India (DPI) and two pigments-burnt sienna pigment (FI-208 Factorizing) and, cobalt blue pigment (FI-203, Factor II, Inc).

\section{Fabrication of Specimens}

Forty specimens for silicone material and 40 specimens for acrylic were fabricated (Fig. 1). The specimens were $2 \mathrm{~mm}$ thick and $20 \mathrm{~mm}$ diameter. Two pigments were added to both the groups.

\section{Grouping of Specimens}

- A: Burnt sienna pigment

- B: Cobalt blue pigment

- Group I-10:1 ratio of base to catalyst silicone elastomer along with $0.2 \%$ burnt sienna pigment were mixed using stainless steel spatula on a glass slab until homogeneous color of the mixture was obtained and packed in the prepared mold. The same procedure was used to fabricate the specimens of silicone elastomer with addition of $0.2 \%$ by weight of cobalt blue pigment.

Group I (Silicon) was further divided into four subgroups with ten specimens each:

- Group IA: Silicone elastomer with burnt sienna not subjected to weathering (control).

- Group IA1: Silicone elastomer with burnt sienna subjected to weathering (weathering).

- Group IB: Silicone elastomer with cobalt blue not subjected to weathering (control).

- Group IB1: Silicone elastomer with cobalt blue subjected to weathering (weathering).

- Group II (heat-cure acrylic): Heat-cure clear acrylic resin polymer and monomer (3:1 ratio) with addition
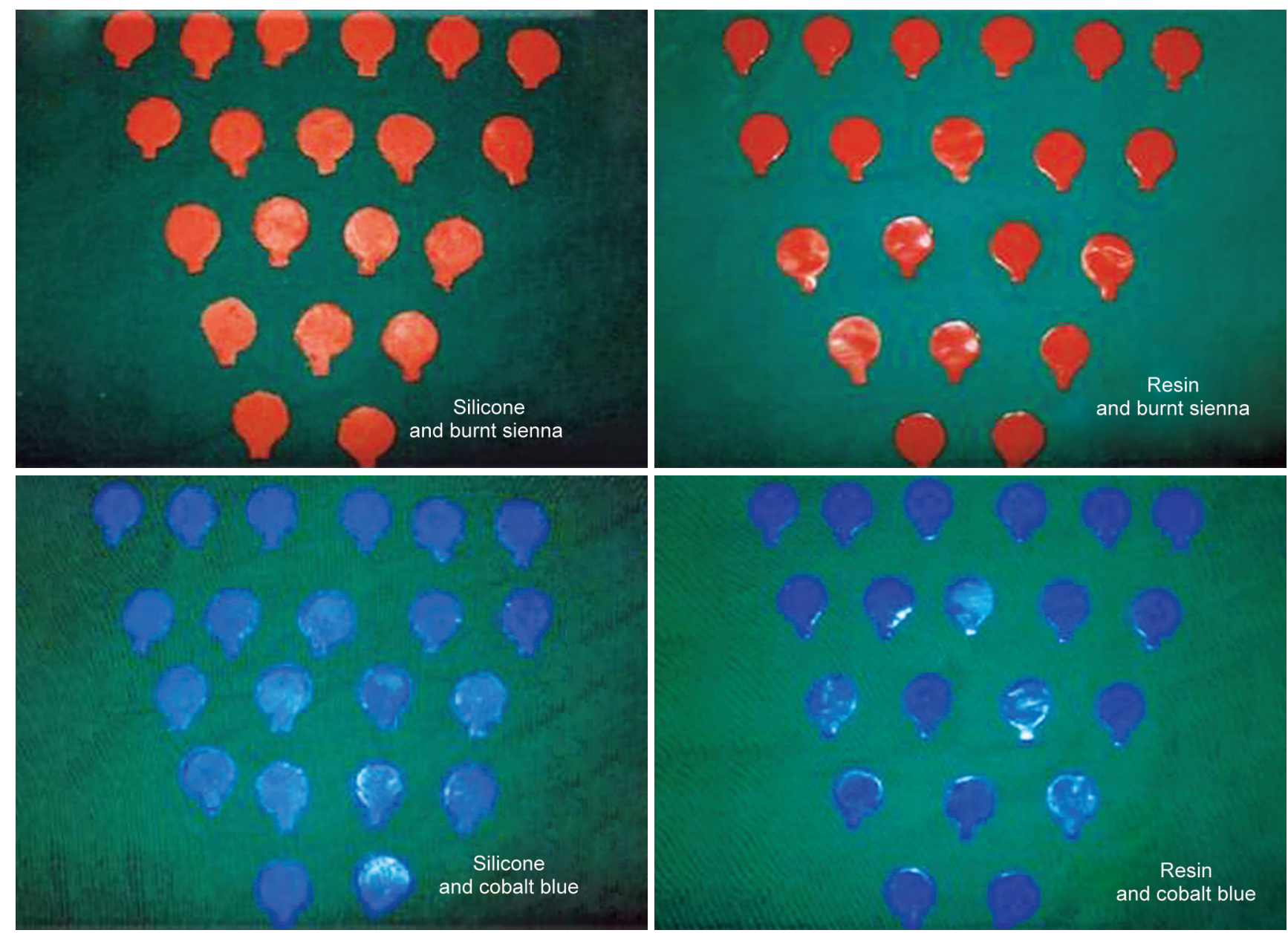

Fig. 1: Specimens of silicone and heat-cure clear acrylic resin mixed with burnt sienna and cobalt blue pigment 
of burnt sienna $0.2 \%$ was mixed and was packed into the prepared mold and subjected to short-term polymerization in a water bath at $72^{\circ}$ for 1.5 hours. The same procedure was carried out for the fabrication of heat-cure acrylic resin specimen with the addition of $0.2 \%$ by weight of cobalt blue pigment.

Group II was further divided into four subgroups with 10 specimens each:

- Group IIA: Heat-cure clear acrylic with burnt sienna not subjected to weathering (control).

- Group IIA1: Heat-cure clear acrylic with burnt sienna subjected to weathering (weathering).

- Group IIB: Heat-cure clear acrylic with cobalt blue not subjected to weathering (control).

- Group IIB1: Heat-cure clear acrylic with cobalt blue subjected to weathering (weathering).

Spectrophotometer (Fig. 2) was calibrated with a standard white card with $L^{*}$ value of $95.5, a^{*}-0.4$ and $b^{*}-2.1$, observer's angle of $2^{\circ}$ and illumination of D50. The values of $L^{*}, a^{*}, b^{*}$ for each specimen before weathering were recorded with white card as the background and were tabulated. Color measurements were made in five selected areas of specimen and mean is calculated using CIE Lab uniform color scale. After base line readings, control specimens were kept in a black box to prevent exposure to outdoor weather. To simulate weathering condition, the environmental chamber (Fig. 3) was used where in the test group was kept for 30 days, at a temperature of $32^{\circ} \mathrm{C}$ during day time and exposed to ultraviolet (UV)-visible light spectrum with a $2500 \mathrm{~W}$ xenon lamp during day time. Lamp was kept off during night. The obtained data were subjected to statistical analysis.

\section{RESULTS}

The present in vitro study was conducted to evaluate the effect of outdoor weathering on color stability of silicone and acrylic resin with pigments. Pairwise comparison (student ' $t$ ' test) of mean of color change $\left(\Delta \mathrm{E}^{*}\right)$ (Table 1 ) shows the mean and standard deviation of color change values obtained for test and control groups. From the p-value, it is evident that four groups differed significantly with respect to their color change. It means that group IA1 had higher color change and group IIB1 had least color change among the four weathering groups.

The results of Scheffe's multiple comparison tests (Table 2) showed that color change of study group IA1 differed significantly from that of other study groups. This is the decreasing order of the color change (Graph 1). This indicates that the color changes of study group

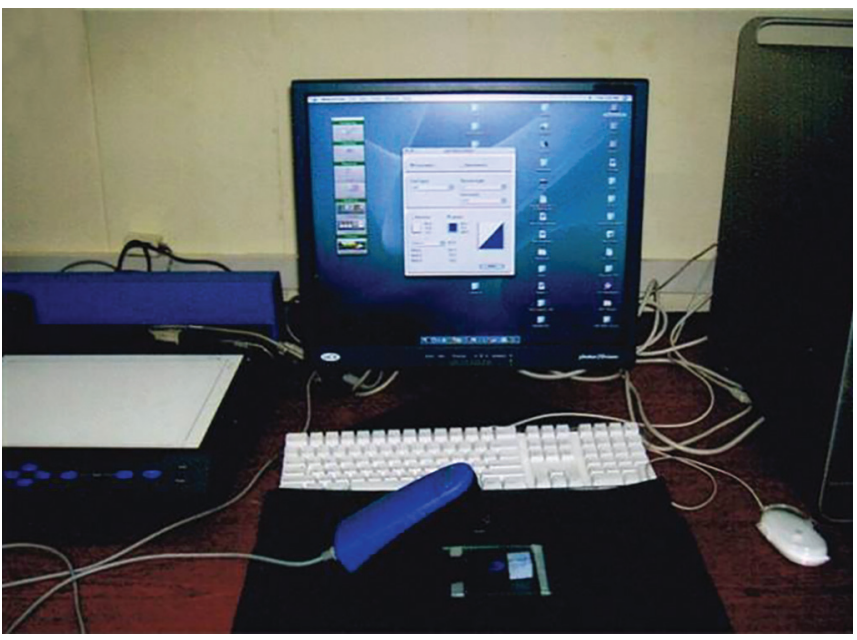

Fig. 2: Spectrophotometer and PC showing CIE Lab values of specimen

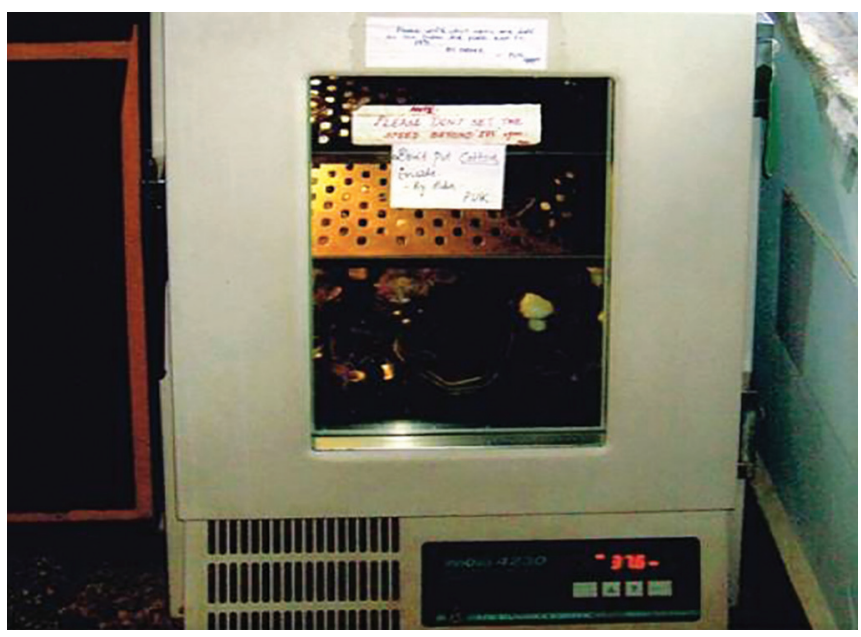

Fig. 3: Environmental chamber with temperature control used for weathering study group specimens of silicone and heat-cure clear acrylic resin

Table 1: Pairwise comparison (student 't' test) of mean of color change $\left(\Delta \mathrm{E}^{*}\right)$

\begin{tabular}{llllll}
\hline Groups & Mean & Std. dev. & t-value & $p$-value & Significance \\
\hline IA & 1.314 & 0.854 & & & \\
IA1 & 11.927 & 1.305 & -21.516 & 0.000 & $\mathrm{~S}$ \\
IB & 3.105 & 1.202 & & & \\
IB1 & 9.481 & 0.958 & -13.111 & 0.000 & $\mathrm{~S}$ \\
IIA & 1.744 & 0.702 & & & \\
IIA1 & 3.220 & 0.868 & -4.178 & 0.0006 & $\mathrm{~S}$ \\
IIB & 4.870 & 1.810 & & & \\
IIB1 & 8.128 & 1.028 & -4.947 & 0.0001 & $\mathrm{~S}$ \\
\hline
\end{tabular}

S: Significant

Table 2: Scheffe's multiple comparison test

\begin{tabular}{lllll}
\hline $\begin{array}{l}\text { Sub } \\
\text { groups }\end{array}$ & IA1 & IB1 & IIB1 & IIA1 \\
\hline Mean & 11.927 & 9.481 & 3.227 & 8.128 \\
IA1 & - & - & - & - \\
IB1 & 0.0001 & - & - & - \\
IIB1 & 0.000 & 0.000 & - & - \\
IIA1 & 0.000 & 0.056 & 0.000 & - \\
\hline
\end{tabular}

L*: Is lightness (white-black); $a^{*}$ and $b^{*}$ : Is chromatic components; $a^{*}$ : Redness to greenness; $b^{*}$ : Yellowness to blueness 


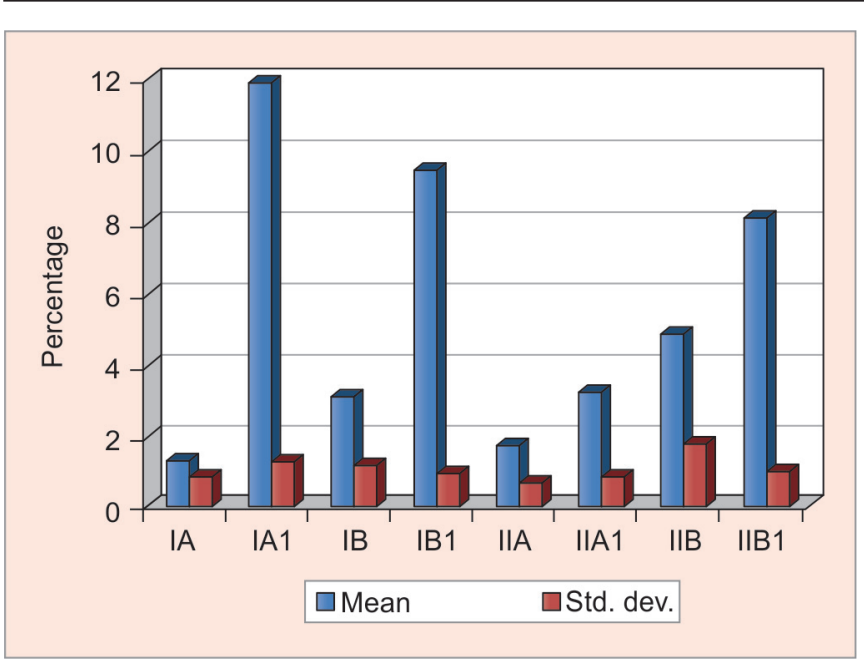

Graph 1: Mean and standard deviation of color change $\left(\Delta \mathrm{E}^{\star}\right)$

(group IA1, group IB1, group IIB1, group IIA1) with elastomer and burnt sienna (IA1) had more color change and the study group with heat-cure and burnt sienna (IIA1) had least color change when compared to other groups. Silicone and burnt sienna (IA1) showed maximum color change, but heat-cure and burnt sienna (IIA1) were the most color stable.

\section{DISCUSSION}

Discoloration of facial prosthesis observed clinically is a complex phenomenon comprised of several contributing factors that include intrinsic color stability of silicone elastomer, pigments and flocking, loss of extrinsic coloration, personal habits and environmental staining. ${ }^{9,11}$

The most serious problems associated with facial prosthesis are poor durability and loss of esthetics, which have been verified by clinical studies.

Chen et al conducted a study to evaluate the reaction of 138 patients to their facial prosthesis. Color fading was the most frequent response given by the patients for disliking their responses. ${ }^{10}$

Color discoloration of facial prosthesis in clinical situations is mainly due to color loss that occurs in certain UV susceptible pigment and various staining. ${ }^{11}$

Two types of colorants are available, organic and inorganic. The inorganic colorants are metallic oxides; these molecules are stable as a result of their ionic bond being insoluble particles inorganic pigments absorb and scatter light due to their particle size and refractive indices, they are more opacifying that the dyes which are soluble, have lower refractive indices and, therefore, more translucent. ${ }^{12}$ Therefore, the purpose of the study was to evaluate the effect of outdoor weathering on color stability of silicone and heat-cure acrylic resin with the addition of two different pigments. A study done by Polyzois in 1999 on color stability after outdoor weathering, he reported that duration of exposure and silicone elastomer was significant factor that affected color stability which is similar to our study. ${ }^{8}$

In the CIE Lab system according to Seghi et $\mathrm{al}^{13}$ and Kuehni and Marcus, ${ }^{14}$ a $\Delta \mathrm{E}^{*}$ equal to 1 is considered visually detectable $50 \%$ of the time, where as a $\Delta \mathrm{E}^{*}$ greater than two was used as a baseline and considered to be visually detectable.

Hulterström et al in their study found that addition type of polymer has less color change when compared to condensation type, it could be attributed to high filler content, lower opacity. The result of our study was in accordance with Hulterström et al. ${ }^{12}$

The difference in color change can be attributed to the chemical nature of silicone elastomer and heat-cure clear acrylic resin as well as chemical nature of pigments burnt sienna and cobalt blue. May et al found the residual monomer in acrylic resin could be one explanation for the color changes observed. Other possible reasons for the changes observed could be attributed to colorants added for characterization. The colorants may leach out or change in color on accelerated aging and another possible source of color change may be associated with porosity caused by over heating or insufficient pressure during polymerization. ${ }^{15}$

Gary et al reported that outdoor weathering tests in which documented American Society for Testing and Materials (ASTM) method provided a valid baseline fir future research on color changes in maxillofacial prosthesis. ${ }^{16}$

Gary et al indicated that early color changes in prosthesis may be the result of degradation of certain UV light-susceptible pigments, whereas long-term color shifts may be caused by color changes within elastomer. ${ }^{11}$ Color deterioration of facial prosthesis is clinical situations is mainly due to color losses that occur in certain UVsusceptible pigments and various staining.

\section{CONCLUSION}

From this study, it can be concluded that heat-cure acrylic with burnt sienna was most color stable followed by heat-cure acrylic with cobalt blue. Silicone elastomer with burnt sienna had undergone significant color change after outdoor weathering.

\section{REFERENCES}

1. Haug SP, Andres CJ, Moore KB. Color stability and colorant effect on maxillofacial elastomers. Part III: wethering effect on color. J Prosthet Dent 1999;81(4):431-438.

2. Lewis DH, Fisher TE, Koran A. External maxillofacial prostheses. In: Reese JA, Valega TM, editors. Restorative dental materials: an overview. London: Quintessence 1985;1:214-241.

3. Ma T. Clinical overview of materials for extraoral maxillofacial prosthetics. Materials research in maxillofacial prosthetics. 
In: Setcos JC, editor. Transactions of the Academy of Dental Materials 1992;5:9-20.

4. Andres CJ, Haug SP, Munoz CA, Bernal G. Effects of environmental factors on maxillofacial elastomers: Part Iliterature review. J Prosthet Dent 1992;68(2):327-330.

5. Andres CJ, Haug SP, Brown DT, Bernal G. Effects of environmental factors on maxillofacial elastomers: Part IIreport of survey. J Prosthet Dent 1992;68(3):519-522.

6. Haug SP, Andres CJ, Munoz CA, Okamura M. Effects of environmental factors on maxillofacial elastomers: Part IIIphysical properties. J Prosthet Dent 1992;68(4):644-651.

7. Haug SP, Andres CJ, Munoz CA, Bernal G. Effects of environmental factors on maxillofacial elastomers: Part Woptical properties. J Prosthet Dent 1992;68(5):820-823.

8. Polyzois GL. Color stability of facial silicone prosthetics polymers after outdoor weathering. J Prosthet Dent 1999;82(4): 447-450.

9. Beatty MW, Mahanna GK, Dick K. Color changes in drypigmented maxillofacial elastomer resulting from ultraviolet light exposure. J Prosthet Dent 1995;74(5):493-498.
10. Chen MS, Udagama A, Drane JB. Evaluation of facial prosthesis for head and neck cancer patients. J Prosthet Dent 1981;46:538-544.

11. Gary JJ, Smith CT. Pigments and their application in maxillofacial elastomers: a literature review. J Prosthet Dent 1998;80(2):204-208.

12. Hulterström AK, Ruyter IE. Changes in appearance of silicone elastomers for maxillofacial prostheses as a result of aging. Int J Prosthodont 1999:12(6):498-504.

13. Seghi RR, Hewlett ER, Kim J. Visual and instrumental colorimetric assessments of small color differences on translucent dental porcelain. J Dent Res 1989;68(12):1760-1764.

14. Kuehni RG, Marcus RT. An experiment in visual scaling of small color differences. Color Res Appl 1979;4(2):83-91.

15. May KB, Shotwell JR, Koran A, Wang RF. Color stability: Denture base resins processed with the microwave method. J Prosthet Dent 1996;76(6):581-589.

16. Gary JJ, Huget EF, Powell LD. 'Accelerated color change in a maxillofacial elastomer with and without pigmentation. J Prosthet Dent 2001;85(6):614-620. 